\title{
The effect of a structured model for stroke rehabilitation multi- disciplinary team meetings on functional recovery and productivity: A Phase I/II proof of concept study
}

Clinical Rehabilitation I-6

(C) The Author(s) 2014

Reprints and permissions: sagepub.co.uk/journalsPermissions.nav DOI: |0.|I77/02692/55|456259| cre.sagepub.com

(SAGE

\section{Sarah F Tyson', Louisa Burton ${ }^{1,2}$ and Alison McGovern²}

\begin{abstract}
Objective: Regular multidisciplinary team meetings are the main way that teams operate, yet our earlier research found they can sometimes be suboptimal. We developed a model to structure multidisciplinary team meetings and assessed the feasibility, acceptability and impact of its implementation on meeting quality and patient outcomes.

Design: Longitudinal cohort design with non-participant observation of multidisciplinary team meetings before and after implementation of the intervention.

Setting: Inpatient stroke rehabilitation units.

Subjects: Members of the multidisciplinary inpatient stroke rehabilitation teams.

Intervention: A model to structure multidisciplinary team meetings.

Main measures: Quality of multidisciplinary team meetings (using a predefined checklist); change in independence (Barthel Index) during admission; length of stay; meeting duration and the number of patients discussed.

Results: At baseline, meeting quality was generally low. Following implementation, all aspects of meeting quality improved by $5 \%-58 \%$. This was achieved without loss of staff productivity or additional resources: The mean number of patients treated during the observation periods was 36 (SD 17.6), which was unchanged after implementation. Nor were there any significant changes in the length of meetings (mean $=76$ minutes), time spent discussing each patient (5.4 vs. 7 minutes) or length of stay (26.7 vs. 30.3 days), but there was a greater increase in Barthel Index score after implementation (3.8 vs. 4.7 ) indicating greater functional recovery.

Conclusions: A feasible and acceptable model to structure multidisciplinary stroke team meetings has been developed and implemented. This increased meeting quality without increase in resources and may increase patient recovery.
\end{abstract}

'Stroke Research Centre School of Nursing, Midwifery \& Social Work, University of Manchester, Manchester, UK ${ }^{2}$ Greater Manchester Cardiac \& Stroke Network, Manchester, UK

\section{Corresponding author:}

Sarah F Tyson, School of Nursing, Midwifery \& Social Work, Jean McFarlane Building, University of Manchester, Oxford Road, Manchester, MI3 9PL, UK.

Email: Sarah.tyson@manchester.ac.uk. 


\section{Keywords}

Rehabilitation, multidiscipliary team, stroke, meetings

Date received: 22 August 20I4; accepted: 9 November 2014

\section{Introduction}

Multidisciplinary teams are the recommended model of service delivery for stroke care ${ }^{1}$ as they contribute to the superiority of organised specialist stroke care over generalist services. ${ }^{2}$ However, although qualitative literature indicates an effective multidisciplinary team can enhance decision-making, co-ordination of care and team climate, objective evidence of the impact of a multidisciplinary team on service quality or patient outcomes is limited. ${ }^{3-7}$ Regular meetings (usually weekly) of the multidisciplinary team to discuss patients' progress and plan treatment are the main process by which multidisciplinary teams work, ${ }^{1}$ but our earlier work has shown the way team meetings are delivered is highly varied in clinical practice and sometimes ineffective. ${ }^{8}$ To address this issue we sought to develop a structured model for multidisciplinary team meetings in stroke rehabilitation (called the Manchester Multi-disciplinary Meeting Model (M4)) and to make an initial (Phase I/II) evaluation of its effect on meeting quality and patient outcomes.

\section{Method}

\section{Development of the M4 model for rehabilitation meetings}

A framework for stroke multidisciplinary team meetings and national guidelines for multidisciplinary team meetings in cancer care were used to identify key elements of a successful multidisciplinary team meeting. ${ }^{3,8,9}$ These were:

- a suitable venue;

- punctual attendance;

- a formalised structure, including use of standardised objective measurement tools and documentation;

- preparation of information to be exchanged;
- progress monitoring;

- action planning;

- effective chairing.

The model to address these elements involved the following.

- A formalised agenda for the discussion of each patient including:

- a brief introduction;

- a review of previous actions (for on-going patients);

- an assessment of patients' impairments and activity limitations (in subsequent weeks, this becomes a review of progress) using standardised measurement tools;

- a statement of patients' goals (in subsequent weeks this becomes a review of progress);

- plans for management or treatment with revisions and updates as necessary;

- planning towards discharge (expected date and destination of discharge to be recorded at initial assessment and reviewed at subsequent meetings);

- identification of actions to be completed with responsibility allocated to a specific team member with date for completion.

- Standardised documentation to support and record the discussion.

- A toolkit of standardised measurement tools (called the Greater Manchester Assessment of Stroke Rehabilitation, G-MASTER) to define the patients' impairments and activity limitations. The specific development and evaluation of the G-MASTER toolkit is reported elsewhere. ${ }^{10}$

- Guidelines for the conduct of meetings, including expectations, preparation, communication, feedback to the wider team and the Chair's role. 
This model was named the Manchester Multidisciplinary Team Meeting Model, or M4. The handbook containing full details can be downloaded from https://www.escholar.manchester.ac.uk/ uk-ac-man-scw:230101.

We worked with stroke rehabilitation units to develop the model, pilot test elements for 'fitness for purpose' in their meetings and, where necessary, accommodate local policies. For example, one site used electronic records and so an electronic version was produced. At each site, a senior member of the multidisciplinary team acted as the 'project champion' who led local development and implementation at each site. Following production of the final prototype, the M4 was implemented, with support from the project champion and authors into all multidisciplinary team meetings using process mapping, 'Plan Do Study Act' cycles, learning sets and action planning. ${ }^{11}$

\section{A cohort evaluation of the M4 model for rehabilitation meetings}

A cohort design was used to evaluate the impact of the M4 model for rehabilitation meetings by comparing the quality of multidisciplinary team meetings, productivity and patient outcomes for three months before and after implementation. Inclusion criteria were minimal; we invited all the stroke rehabilitation units $(n=10)$ in a large city in the UK to participate. Two sites participated in the development and implementation of the model, but declined to participate in the data collection phases. One because they felt the data was too sensitive to be recorded and the other because they did not hold a regular multidisciplinary team meeting. Although we sought ethical approval from the local committee of the National Research Ethics Service, their view was that the project did not require ethical approval. All participating staff gave consent for data collection.

Meeting quality was assessed against a comprehensive checklist of standards produced during the development phase. These consisted of 84 standards grouped into nine elements:

- the venue;

- attendance and punctuality;
- meeting structure and organisation;

- preparation;

- chairing;

- information exchange;

- use of standardised measurement tools;

- monitoring progress;

- action planning.

Full details of the standards are found in Appendix 1 (available online) except the standards for 'the Use of Standardised Measures', which are reported elsewhere. ${ }^{10}$

Performance during the meetings was assessed against these standards using non-participant observation. One of the authors (LB) attended at least two meetings at each site during the baseline and evaluation periods (detailed below). Each meeting was audio-recorded (transcribed verbatim and anonymised), field notes were taken during the meeting, analytic memos made immediately afterwards, and a standardised form completed noting details of the venue and availability of resources. Information that could not be gleaned from the meeting (such as details of preparation and feedback) was obtained from the staff after the meeting. The baseline and evaluation meeting transcripts, field notes and memos from each site were reviewed by the authors and used to score each teams' performance against the standards using the following system.

- $0=$ rarely or never happens.

- 1 = happens but not consistently.

- 2 = happens routinely.

The scores for each item summed to generate a score for all sites. The maximum score for individual items was $16(2 \times 8$, as there were eight participating sites $)$. Then the scores for each item were summed to generate an overall score for each of the nine elements. To enable comparison between elements, a percentage completion rate calculated from the:

score for the element/maximum score for the element (i.e. the number of items $\times 16$ ) $\times 100$

A score of $100 \%$ would indicate that all of the items in that element were met, in all participating sites. 
Productivity was measured by calculating the mean length of time spent discussing each patient, meeting duration and the number of patients discussed in each meeting. Impact on patient outcomes was assessed by measuring length of hospital stay and change in independence in the activities of daily living (Barthel Index) between admission and discharge. These data were collected for all patients discharged from each stroke unit for three months before and three months after implementation. It was gathered by the clinical teams from patients' clinical notes using their standard activity recording processes. As the meeting model was designed for use in stroke rehabilitation, we only sought data for patients receiving rehabilitation (and some of the rehabilitation units were combined with acute care). Thus we excluded patients who were not considered candidates for rehabilitation. This was defined as: patients who died; had an admission Barthel Index score of 20 (indicating they were independent on admission); and/or a stay of less than seven days.

Descriptive statistics and Mann-Whitney $U$-tests were used to describe and assess changes in meeting performance, while independent $t$-tests compared productivity, length of stay and changes in Barthel Index score.

\section{Results}

The participating stroke rehabilitation units and their multidisciplinary team meetings have been described in detail previously. ${ }^{8}$ In summary, four units provided a combined acute care and rehabilitation service and four were 'stand-alone' rehabilitation units. All sites held well-established, multidisciplinary team meetings at least weekly. In these, the overall aim was to share information and come to joint decisions regarding patients' problems, their progress and treatment plans. Three sites also had a separate meeting to address patients' goals involving therapists \pm nurses. Two sites also held nurse-led daily ward meetings with the therapists to follow up actions and note changes in patient care. Between seven and 28 patients were discussed in the meetings. In total, the discussions on 242 stroke patients were recorded and evaluated;
118 before implementation of the M4 model of rehabilitation meetings and 124 patients after implementation.

Baseline data indicated that meeting quality was generally low before implementation of the M4 model for rehabilitation meetings. All elements except 'the venue' and 'chairing skills' scored below 50\% (Table 1). 'Monitoring progress' and 'planning actions' (which includes decision making) were particularly ineffectively addressed (scoring $28 \%$ and $17 \%$, respectively).

Following implementation of the meeting model, performance in all elements of meeting quality improved ( $p=0.000$ to 0.002 , Table 1) except 'the venue', which increased insignificantly from $86 \%$ to $91 \%$. After implementing the meeting model, meeting quality rose to at least $75 \%$ for all elements except 'monitoring progress' and 'use of standardised measurement tools'. Although these elements improved significantly, the scores were still suboptimal after implementation at 53\% and $51 \%$, respectively. Details of the change in each individual standard are available from the authors.

The changes in meeting quality were achieved without loss of staff productivity or additional resources and suggested that patients had greater functional recovery (Table 2). The mean number of patients treated during the observation periods was 36 (SD 17.6, range 16 to 74), with no change after implementation $(p=0.44)$, nor were there any differences in the length of meetings $(p=1)$, time spent discussing each patient $(p=0.192)$ or length of stay ( $p=0.073$, Table 2$)$, but there was a greater increase in Barthel Index score after implementation indicating greater functional recovery $(p=0.038)$.

\section{Discussion}

The results of this phase I/II cohort study indicate that a structured model for multidisciplinary team meetings can feasibly and acceptably be implemented. Furthermore, it may have a beneficial effect on multidisciplinary team function and patients' functional recovery without additional resources. Although multidisciplinary teamworking is the globally recommended model to deliver 
Table I. Meeting performance before and after implementation of a structured meeting model.

\begin{tabular}{|c|c|c|c|}
\hline & $\begin{array}{l}\text { Performance before } \\
\text { implementation }\end{array}$ & $\begin{array}{l}\text { Performance after } \\
\text { implementation }\end{array}$ & $\begin{array}{l}P \text { value for } \\
\text { comparison }\end{array}$ \\
\hline Venue & $69 / 80(86 \%)$ & $73 / 80(91 \%)$ & 0.492 \\
\hline \multicolumn{4}{|l|}{ No of items $=5 ;$ maximum score $=80$} \\
\hline Attendance \& punctuality & $82 / 176(47 \%)$ & 136/176 (77\%) & 0.000 \\
\hline \multicolumn{4}{|l|}{ No of items $=11 ;$ maximum score $=176$} \\
\hline Meeting structure \& organisation & $38 / 96(48 \%)$ & $83 / 96(86 \%)$ & 0.002 \\
\hline \multicolumn{4}{|l|}{ No of items $=6 ;$ maximum score $=96$} \\
\hline Preparation & $40 / 80(50 \%)$ & $70 / 80(88 \%)$ & 0.001 \\
\hline \multicolumn{4}{|l|}{ No of items $=5 ;$ maximum score $=80$} \\
\hline Use of standardised measurement tools & $161 / 416(39 \%)$ & $213 / 416(51 \%)$ & 0.000 \\
\hline \multicolumn{4}{|l|}{ No of items $=26 ;$ maximum score $=416$} \\
\hline Information exchange & $46 / 112$ (4I\%) & $89 / 112$ (79\%) & 0.000 \\
\hline \multicolumn{4}{|l|}{ No of items $=7 ;$ maximum score $=112$} \\
\hline Chairing skills & $101 / 160(63 \%)$ & |46/I60 (9l\%) & 0.001 \\
\hline \multicolumn{4}{|l|}{ No of items $=10 ;$ maximum score $=160$} \\
\hline Monitoring progress & $31 / 112(28 \%)$ & $6 \mathrm{I} / \mathrm{I} / 2(53 \%)$ & 0.001 \\
\hline \multicolumn{4}{|l|}{ No of items $=7 ;$ maximum score $=112$} \\
\hline Action planning & 16/96 (17\%) & $72 / 96(75 \%)$ & 0.000 \\
\hline No of items $=6 ;$ maximum score $=96$ & & & \\
\hline
\end{tabular}

Table 2. Productivity before and after implementation of a structured model for multidisciplinary team meetings.

\begin{tabular}{llll}
\hline & $\begin{array}{l}\text { Productivity } \\
\text { before M4 }\end{array}$ & $\begin{array}{l}\text { Productivity after } \\
\text { M4 }\end{array}$ & $\begin{array}{l}\text { P value for } \\
\text { comparison }\end{array}$ \\
\hline $\begin{array}{l}\text { Mean (SD, range) length of } \\
\text { meeting (minutes) }\end{array}$ & $76(28,60-145)$ & $76(23,35-120)$ & I $(-27,27)$ \\
$\begin{array}{l}\text { Mean (SD, range) time discussing } \\
\text { each patient (minutes) }\end{array}$ & $5.4(1.7,3-7)$ & $7(3,4-10)$ & $0.195(-4,0.99)$ \\
$\begin{array}{l}\text { Patient throughput during the } \\
\text { observation period }\end{array}$ & $32.3(14.7)$ & $39.9(20.6)$ & $0.44(-28.40,13.26)$ \\
$\begin{array}{l}\text { Mean (SD) length of stay (days) } \\
\text { Mean (SD) change in Barthel Index }\end{array}$ & $26.7(22.5)$ & $30.3(29.3)$ & $0.073(-7.42,0.33)$. \\
\hline
\end{tabular}

M4: Manchester Multi-disciplinary Meeting Model.

stroke care and multidisciplinary team meetings are considered a key element of successful teamwork and effective specialist services, ${ }^{1,2}$ this it is a notably under-researched area. To the authors' knowledge, this is the first study to report an intervention to improve function of multidisciplinary stroke teams.

Despite the encouraging results, the results need to be treated with caution as the design used is open to bias. The uncontrolled design means the observed changes cannot be presumed to be solely owing to implementation of the meeting model. The study was undertaken during a period of health service re-organisation and other changes in practice may have had an influence. However, the degree of observed improvement and the difficulty teams were experiencing before implementation makes this an unlikely explanation for the observed 
changes during the meetings. Other changes in practice may have influenced patient outcomes however. Furthermore data collection was unblinded and staff knew they were being assessed, so detection bias and a Hawthorne effect may have been present. Thus, the results do not indicate that the meeting model was effective but that it has potential to be so. Randomized controlled trials to evaluate more robustly the impact of the meeting model on service delivery and outcomes are warranted. Qualitative work considering staff's experience and views of the impact of M4 could elucidate potential mechanisms for the changes seen.

Generalisability of the findings to other centres should be treated cautiously, as the model was designed for inpatient rehabilitation in the UK healthcare system; it may not be as applicable in other healthcare settings or systems. However, we observed the multidisciplinary team discussions of several hundred stroke patients and involved the stroke units in one of the UK's largest conurbations that employs a broad range of service delivery models and serves a highly varied population of over 3 million people. Thus we feel the findings are reasonably representative of inpatient stroke rehabilitation. Further work to explore the use of the meeting model in other clinical areas and other settings are planned.

\section{Clinical message}

- A structured model for multidisciplinary team meetings in patient stroke rehabilitation can be feasibly and acceptably implemented and could improve patient outcomes without additional resource.

\section{Conflict of interest}

The authors declare that there is no conflict of interest.

\section{Funding}

The project was funded by the UK Knowledge Transfer Partnership supported by the Technology Strategy Board, and Greater Manchester Cardiac \& Stroke Network [grant number 0007812].

\section{References}

1. Intercollegiate Stroke Working Party. National clinical guideline for stroke. 4th ed. London: Royal College of Physicians, 2012.

2. Langhorne, Pollock in conjunction with the Stroke Unit Trialists' Collaboration. What are the components of effective stroke unit care? Age Ageing 2002; 31(5): 365-371.

3. Harris R, Sims S, Hewitt J, et al. (2013) Inter-professional teamwork across stroke care pathways: outcomes and patient and carer experience. Final report. NIHR Service Delivery and Organisation Programme. Southampton, UK: Her Majesty's Stationery Office.

4. Michan S and Rodger S. Characteristics of effective teams: a literature review. Aust Health Rev 2000: 23; 201208.

5. Fleissig A, Jenkins V, Catt S, et al. Multi-disciplinary teams in cancer care: are they effective in the UK? Lancet Oncol 2006; 7: 935-943.

6. Strasser DC, Falconer JA, Herrin JS, et al. Team functioning and patient outcomes in stroke rehabilitation. Arch Phys Med Rehabil 2005; 86: 403-409.

7. Strasser DC, Falconer JA, Stevens AB, et al. Team training and stroke rehabilitation outcomes: a cluster randomized trial. Arch Phys Med Rehabil 2008; 89: 10-15.

8. Tyson S, Burton L and McGovern A. Multi-disciplinary team meetings in stroke rehabilitation: An observation study. Clin Rehab. Epub ahead of print 4 June 2014. DOI: 10.1177/0269215514535942.

9. Taylor C and Ramirez AJ. Multi-disciplinary team members' views about multi-disciplinary team working: results from a survey commissioned by the National Cancer Action Team. NHS National Cancer Action Team, 2009.

10. Tyson S, Burton L and McGovern A. The impact of an assessment toolkit on use of objective measurement tools in stroke rehabilitation. Clin Rehab. Epub ahead of print 2014. DOI: $10.1177 / 0269215514562590$.

11. Nakayama DK, Bushey TN, Hubbard I, et al. Using a plan-do-study-act cycle to introduce a new OR service line. AORN J 2010; 92; 335-343. 